\section{Brewster Condition for Planar Interfaces of Natural Optically Active Media}

\section{Akhlesh Lakhtakia \\ Department of Engineering Science and Mechanics, The Pennsylvania State University, University Park, PA 16802-1484, USA}

Z. Naturforsch. 47 a, 921-922 (1992); received April 25, 1991

Reflection of planewaves at the planar interface of two natural optically active media has been examined in order to obtain the Brewster condition.

Sir David Brewster published [1] in 1815 the results of his experiments on the reflection of unpolarized light from planar dielectric-dielectric interfaces; his work gave rise to the Brewster angle and was condensed by him into the Brewster law: If unpolarized light is incident under this angle, the reflected light is plane-polarized. During the 1950s, however, it appears [2] that the original definition of the Brewster angle as the polarizing angle was superseded by a new one: a zero-reflection angle. In my opinion, the increasing use of complex (but homogeneous) materials strongly suggests a return to the original definition, and even a broadening of the concept behind the Brewster angle.

Plane waves propagating towards (or away from) a planar interface can be expressed in terms of two distinct and orthogonal eigenmodes [3]. It is conjectured that a condition may exist when the ratio of the amplitudes of the two eigenmodes of the reflected wave is independent of the ratio of the amplitudes of the two eigenmodes of the incident wave.

This condition, to be called after Brewster, may be easily quantified in terms of the horizontal wavenumber $x$ that comes in as a consequence of Snel's laws. For the fulfilment of the Brewster condition, the value of the wavenumber $x$ depends on the relative properties of the two homogeneous media occupying either side of the planar interface. This conjecture has been tested for the planar interfaces of (i) a natural optically active [4] and an isotropic dielectric-magnetic medium

Reprint requests to Prof. A. Lakhtakia, Department of Engineering Science and Mechanics, The Pennsylvania State University, 227 Hammond Building, University Park, PA 16802-1484, USA.
[5], (ii) a natural optically active and an uniaxial dielectric medium [6], and (iii) an isotropic dielectricmagnetic and a general uniaxial medium [7]. In this brief communication, the conjecture will be shown to hold true for the planar interfaces of two natural optically active media (chiral) also.

Consider the material interface $z=0$. The half-space $z \geq 0$ is filled with the homogeneous, chiral medium characterized by

$$
\mathbf{D}=\varepsilon_{a} \mathbf{E}+\varepsilon_{a} \beta_{a} \nabla \times \mathbf{E}, \quad \mathbf{B}=\mu_{a} \mathbf{H}+\mu_{a} \beta_{a} \nabla+\mathbf{H} ;
$$

while the half-space $z \leq 0$ is filled with another chiral medium $\left[\mathbf{D}=\varepsilon_{b} \mathbf{E}+\varepsilon_{b} \beta_{b} \nabla \times \mathbf{E}, \mathbf{B}=\mu_{b} \mathbf{H}+\mu_{b} \beta_{b} \nabla \times \mathbf{H}\right]$. An $\exp [-i \omega t]$ time-dependence is implicit thoroughout; the wavenumbers

$$
\begin{array}{ll}
\gamma_{1 a}=k_{a} /\left(1-k_{a} \beta_{a}\right), & \gamma_{2 a}=k_{a} /\left(1+k_{a} \beta_{a}\right), \\
\gamma_{1 b}=k_{b} /\left(1-k_{b} \beta_{b}\right), & \gamma_{2 b}=k_{b} /\left(1+k_{b} \beta_{b}\right)
\end{array}
$$

are defined for the two media, with $k_{a, b}=\omega \sqrt{\left(\mu_{a, b} \varepsilon_{a, b}\right)}$ being shorthand notations; and the impedances are given by $\eta_{a, b}=\sqrt{\left(\mu_{a, b} / \varepsilon_{a, b}\right)}$.

In a natural optically active medium, the electromagnetic fields, $\mathbf{E}$ and $\mathbf{H}$, can be expressed in terms of Beltrami fields $\mathbf{Q}_{1}$ and $\mathbf{Q}_{2}[4,8]$; thus,

$$
\begin{aligned}
& \mathbf{E}_{a, b}=\mathbf{Q}_{1 a, b}-i \eta_{a, b} \mathbf{Q}_{2 a, b} \text { and } \\
& \mathbf{H}_{a, b}=\mathbf{Q}_{2 a, b}-\left(i / \eta_{a, b}\right) \mathbf{Q}_{1 a, b}
\end{aligned}
$$

in the two regions. Without loss of generality, let $z \geq 0$ be the zone of incidence and reflection, while the zone $z \leq 0$ be that of transmission. Consequently the planewave representation for the two chiral media can be set up as $[5,6]$

$$
\begin{array}{r}
\mathbf{Q}_{1 a}=A_{1}\left[\mathbf{e}_{y}+i\left(\alpha_{1 a} \mathbf{e}_{x}+\chi \mathbf{e}_{z}\right) / \gamma_{1 a}\right] \exp \left[i\left(\varkappa x-\alpha_{1 a} z\right)\right] \\
+R_{1}\left[\mathbf{e}_{y}+i\left(-\alpha_{1 a} \mathbf{e}_{x}+\chi \mathbf{e}_{z}\right) / \gamma_{1 a}\right] \exp \left[i\left(\chi x+\alpha_{1 a} z\right)\right] ; \\
z \geq 0, \quad(1 \mathrm{a}) \\
\mathbf{Q}_{2 a}=A_{2}\left[\mathbf{e}_{y}-i\left(\alpha_{2 a} \mathbf{e}_{x}+\chi \mathbf{e}_{z}\right) / \gamma_{2 a}\right] \exp \left[i\left(\varkappa x-\alpha_{2 a} z\right)\right] \\
+R_{2}\left[\mathbf{e}_{y}-i\left(-\alpha_{2 a} \mathbf{e}_{x}+\chi \mathbf{e}_{z}\right) / \gamma_{2 a}\right] \exp \left[i\left(\chi x+\alpha_{2 a} z\right)\right] ; \\
z \geq 0, \quad(1 \mathrm{~b}) \\
\mathbf{Q}_{1 b}=T_{1}\left[\mathbf{e}_{y}+i\left(\alpha_{1 b} \mathbf{e}_{x}+\chi \mathbf{e}_{z}\right) / \gamma_{1 b}\right] \exp \left[i\left(\chi x-\alpha_{1 b} z\right)\right] ; \\
z \leq 0, \quad(1 \mathrm{c}) \\
\mathbf{Q}_{2 b}=T_{2}\left[\mathbf{e}_{y}-i\left(\alpha_{2 b} \mathbf{e}_{x}+\chi \mathbf{e}_{z}\right) / \gamma_{2 b}\right] \exp \left[i\left(\varkappa x-\alpha_{2 b} z\right)\right] ; \\
z \leq 0, \quad(1 \mathrm{~d})
\end{array}
$$

0932-0784 / 92 / 0700-0921 \$01.30/0. - Please order a reprint rather than making your own copy. 
The coefficients $A_{1}$ and $A_{2}$ are the complex amplitudes of the planewave eigenmodes incident on the interface, $R_{1}$ and $R_{2}$, of the planewave eigenmodes reflected off the interface into the zone $z \geq 0$; while $T_{1}$ and $T_{2}$ are for the planewave eigenmodes transmitted into the zone $z \leq 0$. Finally, $x$ is the horizontal wavenumber required by Snel's law to satisfy the phasematching condition of the interface $z=0$;

$$
\begin{aligned}
& \alpha_{1 a, b}=+\sqrt{\left(\gamma_{1 a, b}^{2}-\varkappa^{2}\right)} \text { and } \\
& \alpha_{2 a, b}=+\sqrt{\left(\gamma_{2 a, b}^{2}-\varkappa^{2}\right)},
\end{aligned}
$$

and $\mathbf{e}_{x}, \mathbf{e}_{y}$, and $\mathbf{e}_{z}$ are the unit Cartesian vectors.

The boundary value problem is solved by ensuring the continuity of the tangential components of the $\mathbf{E}$ and the $\mathbf{H}$ fields across the interface $z=0$. For a given $x$, the resulting solution is best stated in matrix notation as follows:

$\left(\begin{array}{l}R_{1} \\ R_{2}\end{array}\right)=\left(\begin{array}{ll}r_{11} & r_{12} \\ r_{21} & r_{22}\end{array}\right)\left(\begin{array}{l}A_{1} \\ A_{2}\end{array}\right), \quad\left(\begin{array}{l}T_{1} \\ T_{2}\end{array}\right)=\left(\begin{array}{ll}t_{11} & t_{12} \\ t_{21} & t_{22}\end{array}\right)\left(\begin{array}{l}A_{1} \\ A_{2}\end{array}\right)$.

The various Fresnel reflection and transmission coefficients involved in the foregoing matrices are given as

$r_{11}=\left(u_{-}+v_{-}\right) /\left(u_{+}+v_{+}\right)$

$r_{12}=2 w \xi_{2 a}\left(i \eta_{a}\right) /\left(u_{+}+v_{+}\right)$,

$r_{12}=2 w \xi_{1 a} /\left(i \eta_{a}\right)\left(u_{+}+v_{+}\right)$,

$r_{22}=\left(u_{-}-v_{-}\right) /\left(u_{+}+v_{+}\right)$,

$t_{11}=4 \eta_{b}\left(\eta_{a}+\eta_{b}\right) \xi_{1 b}\left(\xi_{2 a}+\xi_{2 b}\right) /\left(u_{+}+v_{+}\right)$,

$t_{12}=-4 i \eta_{a} \eta_{b}\left(\eta_{a}-\eta_{b}\right) \xi_{2 a}\left(\xi_{1 a}-\xi_{1 b}\right) /\left(u_{+}+v_{+}\right)$,

$t_{21}=4 i\left(\eta_{a}-\eta_{b}\right) \xi_{1 a}\left(\xi_{2 a}-\xi_{2 b}\right) /\left(u_{+}+v_{+}\right)$,

$t_{22}=4 \eta_{a}\left(\eta_{a}+\eta_{b}\right) \xi_{2 a}\left(\xi_{1 a}+\xi_{1 b}\right) /\left(u_{+}+v_{+}\right)$.
The following functions have been used in $(3 \mathrm{a}-\mathrm{d})$ and $(4 a-d)$ :

$$
\begin{aligned}
& u_{ \pm}=4 \eta_{a} \eta_{b}\left(\xi_{1 a} \xi_{2 a} \pm \xi_{1 b} \xi_{2 b}\right), \\
& w=\left(\eta_{a}^{2}-\eta_{b}^{2}\right)\left(\xi_{1 b}+\xi_{2 b}\right), \\
& v_{ \pm}=\left(\eta_{a}-\eta_{b}\right)^{2}\left(\xi_{1 a} \xi_{1 b} \pm \xi_{2 a} \xi_{2 b}\right) \\
& +\left(\eta_{a}+\eta_{b}\right)^{2}\left(\xi_{1 a} \xi_{2 b} \pm \xi_{2 a} \xi_{1 b}\right), \\
& \xi_{1 a, b}=\alpha_{1 a, b} / \gamma_{1 a, b} \text {, } \\
& \xi_{2 a, b}=\alpha_{2 a, b} / \gamma_{2 a, b} \text {. }
\end{aligned}
$$

In order that the reflection amplitude ratio $\left(R_{1} / R_{2}\right)$ be independent of the incidence amplitude ratio $\left(A_{1} / A_{2}\right)$, the equality

$$
r_{12} r_{21}=r_{11} r_{22}
$$

must be satisfied. For the condition (6) to hold, $\varkappa$ must satisfy the relation $u_{+}=v_{+}$, i.e.,

$$
\begin{aligned}
& 4 \eta_{a} \eta_{b}\left(\xi_{1 a} \xi_{2 a}+\xi_{1 b} \xi_{2 b}\right) \\
& =\left(\eta_{a}-\eta_{b}\right)^{2}\left(\xi_{1 a} \xi_{1 b}+\xi_{2 a} \xi_{2 b}\right) \\
& +\left(\eta_{a}+\eta_{b}\right)^{2}\left(\xi_{1 a} \xi_{2 b}+\xi_{2 a} \xi_{1 b}\right) .
\end{aligned}
$$

It is to be noted that interchanging the symbols $a$ and $b$ in the subscripts of the quantities appearing in (7) does not alter that equation; ergo, (7) broadens the concept of the Brewster law for planar interfaces between two natural optically active materials, regardless of which half-space the incidence is from. Hence, (7) should be called the Brewster condition for chiralchiral interfaces.

The conjecture mentioned at the beginning of this communication, and verified in three earlier instances [5-7], thus holds true for yet another case.

[5] A. Lakhtakia, V. V. Varadan, and V. K. Varadan, J. Opt. Soc. Amer. A 7, 1654 (1990).

[6] A. Lakhtakia, Optik 84, 160 (1990).

[7] A. Lakhtakia, K. V. Varadan, and V. V. Varadan, J. Mod. Opt. 38, 649 (1991).

[8] A. Lakhtakia, Speculat. Sci. Tech. 14, 2 (1991).

[4] A. Lakhtakia, V. K. Varadan, and V. V. Varadan, TimeHarmonic Electromagnetic Fields in Chiral Media, Springer-Verlag, Berlin 1989. 
Radiation Induced Colour and Crystal Defects in Topaz

\section{A. Banerjee}

Institut für Geowissenschaften, Johannes-Gutenberg-Universität, Mainz

Z. Naturforsch. 47 a, 923-924 (1992); received April 15, 1992

Changes of the colour of Topaz on $\gamma$-irradiation and corresponding crystal defect induced changes of X-ray transparency and IR spectra are observed and discussed.

Topaz, an aluminium flourosilicate, has the formula $\mathrm{Al}_{2}\left[\mathrm{~F}_{2} \mid \mathrm{SiO}_{4}\right]$, where $\mathrm{F}$ is generally replaced in varying amounts by $\mathrm{OH}$. Some colourless topaz, when treated with $\gamma$-rays, high energy electrons or neutrons, develop brown and/or blue colour [1]. The present note deals with the distribution of the brown colour caused by $\gamma$-irradiation and the corresponding crystal defects, as revealed by the infrared spectra. Several crystals of colourless Brazilian topaz were treated by $\gamma$-rays, upon which certain parts of the crystals turned brown.

Reprint requests to Dr. A. Barnerjee, Institut für Geowissenschaften, Universität Mainz, W-6500 Mainz, FRG.
The crystals could be devided into two types, according to the distribution of colour. In the first type of crystals the colour was confined in certain mantle zones, running parallel to the prism faces, leaving the central part of the crystal colourless (Figure 1). The colouration in the second type of crystals, on the other hand, was not zonal but quite uniform in one part, while the other part remained colourless; the boundary between the coloured and the colourless region was sharply marked (Figure 2).

$\mathrm{X}$-ray radiograms of $2 \mathrm{~mm}$ thick plates cut perpendicular to the $c$-axis of the crystals showed that the brown coloured areas of the treated crystals had become more transparent to $\mathrm{X}$-ray, due to the radiation induced crystal defects, than the colourless areas, which remained unaffected by irradiation.

In addition to that, the infrared spectra (Fig. 3) of the samples taken from the brown coloured regions differ from those taken from the colourless regions of the crystals, particularly between $3650 \mathrm{~cm}^{-1}$ and $3400 \mathrm{~cm}^{-1}$.

This phenomenon is correlated with the crystal defects produced by irradiation. According to [2] the $\mathrm{Fe}^{2+}$ impurity atoms in topaz are ionized to $\mathrm{Fe}^{3+}$ by the irradiation and the free electrons are localized on

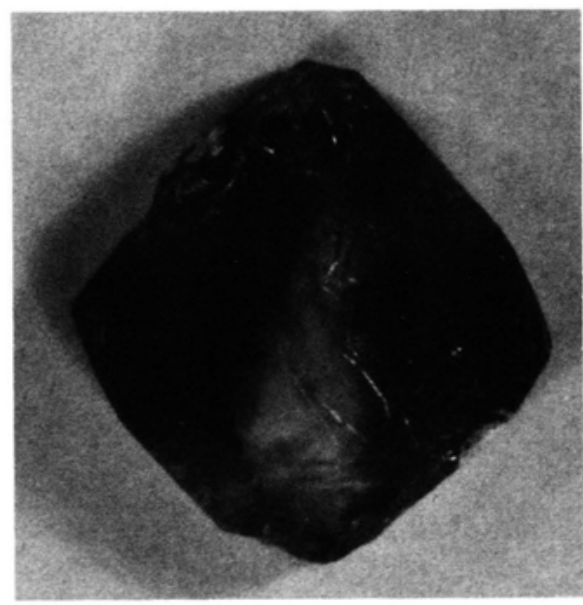

Fig. 1. Effect of $\gamma$-irradiation in a topaz crystal (first type): Crystal plate (001) cut perpendicular to the $c$-axis; mantle zone turned brown, middle part remained colourless.

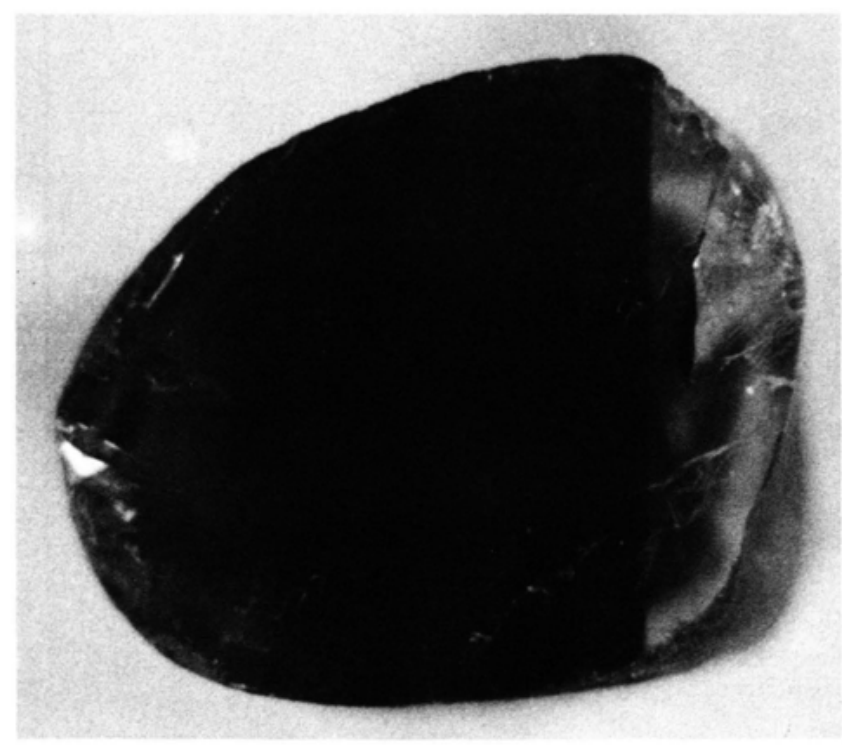

Fig. 2. Effect of $\gamma$-irradiation in topaz (second type): Crystal plate (001) cut perpendicular to the $c$-axis; sharp boundary between the brown and colourless part of the crystal. 
the $(\mathrm{OH})^{-}$vacancies, forming defect centers. It was proposed, that special hydroxide sites, rather than the crystallographically identified ones, correlate with the formation of brown colour centers [3].

$\mathrm{X}$-ray topographic investigation of the treated topaz (in progress) may be helpful in localizing the crystal defects more exactly.

[1] K. Nassau, Gems of Gemology, Spring (1985).

[2] A. N. Platonov and P. Belichenko, Morfol., Svoistav, Genesis Mineralov AN Ukr. SSSR 69 (1965).

[3] R. D. Aines and G. R. Rossmann, Amer. Miner. 71 (1986).

Fig. 3. IR spectra of a topaz after $\gamma$-irradiation. Top: colourless region; below: brown coloured region.

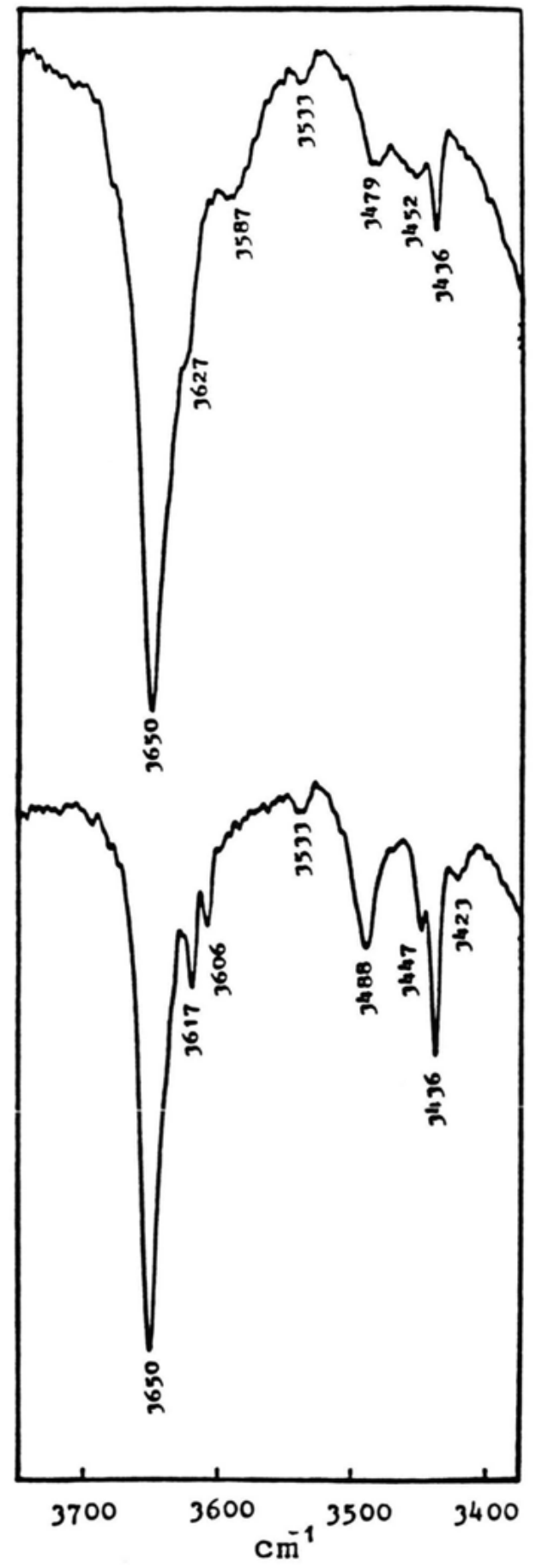

Nachdruck - auch auszugsweise - nur mit schriftlicher Genehmigung des Verlages gestattet

Verantwortlich für den Inhalt: A. KLEMM

Satz und Druck: Konrad Triltsch, Würzburg 